\title{
A Study of Psychomotor Speed and Visuomotor Coordination in Type 2 Diabetes Mellitus
}

\author{
Sandeep Alex ${ }^{1}$ \\ ${ }^{1}$ Department of Psychiatry, Government T. D. Medical College, Alappuzha, Kerala, India.
}

\section{ABSTRACT}

\section{BACKGROUND}

Diabetes mellitus (DM) affects nearly 20\% individuals above 65 years of age. With increasing life expectancy around the world, the absolute number of patients with type 2 diabetes mellitus has seen an exponential increase. Not surprisingly, the macrovascular complications associated with diabetes attracted the attention of clinicians and researchers. However, the cognitive dysfunctions associated with diabetes are studied less. Now, emerging evidence indicates that type 2 diabetes mellitus adversely affects cognitive functions. The studies of cognitive dysfunctions in type 2 diabetes have shown mixed results, with some studies showing a global decline while others reporting more circumscribed deficits in cognitive functions. Against this backdrop, current study set out to determine the effect of type 2 DM on a set of cognitive functions namely attention, sequencing, visuomotor coordination, psychomotor speed and motor persistence. These cognitive functions were chosen because of the possibility that they are more vulnerable to the effects of type $2 \mathrm{DM}$ on brain and are affected earlier.

\section{METHODS}

30 patients with type 2 DM were matched against 30 nondiabetic controls. The sample was matched for age, gender, education and socio-economic status. The tools used were Trail Making Test - Part B (TMT-B) and Digit Symbol Substitution Test (DSST). Data was analysed using Student's t- test, Chi-square test, ANOVA and Mann Whitney U test.

\section{RESULTS}

The mean time taken measured in seconds by patients with type 2 diabetes on TMTB was 264.16 while controls took 144.66 seconds. In DSST, Diabetics took mean time of 531.83 seconds while the control group took mean time of 285.83 seconds to perform the test. The difference between the two groups was very highly significant ( $p$ value 0.001 ). The mean number of errors made by the cases group in TMT-B was 6.86 while those made by the control group was 1.70 . In DSST, the mean number of errors was 8.26 for diabetics and 2.40 for controls. Again, the difference in performance between the two groups was very highly significant ( $p$ value 0.001 ). In patients with diabetes, the duration of illness resulted in very highly significant difference in the time taken for completion and number of errors made in both TMT$\mathrm{B}$ and DSST with people with greater than 10 years of type 2 DM performing poorly than those with less than 5 years of illness (p value 0.001 ).

\section{CONCLUSIONS}

Diabetics had significant cognitive dysfunctions in cognitive domains of attention, sequencing, visual search, visuomotor coordination, motor persistence and response speed with respect to normoglycaemic controls. Furthermore, these deficits increased with longer duration of diabetes and advancing age.

\section{KEY WORDS}

Cognitive Dysfunctions, Type 2 Diabetes Mellitus, HbA1c, TMT-B, DSS

\author{
Corresponding Author: \\ Dr. Sandeep Alex, \\ \#117-E/VII, Anaiah, \\ Whitefield Residence, \\ Kudamaloor, Kottayam-686017, \\ Kerala, India. \\ E-mail:drsandeepalex@yahoo.co.in
}

DOI: $10.14260 / \mathrm{jemds} / 2019 / 848$

Financial or Other Competing Interests: None.

How to Cite This Article:

Alex S. A study of psychomotor speed and visuomotor coordination in type 2 diabetes mellitus. J. Evolution Med. Dent. Sci. 2019;8(52):3915-3919,

$10.14260 /$ jemds $/ 2019 / 848$

Submission 24-10-2019,

Peer Review 12-12-2019,

Acceptance 19-12-2019,

Published 30-12-2019.

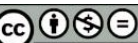




\section{BACKGROUND}

The word diabetes is derived from the Greek diabaine meaning "to pass through" reflecting the copious amount of urine and mellitus is derived from the Latin meaning "sweetened with honey" reflecting the presence of sugar in the urine. ${ }^{1}$

Past 30 years have witnessed an exponential increase in the absolute number of diabetic patients as the life expectancy of humans has increased around the world. Now diabetes mellitus affect nearly $20 \%$ of individuals above 65 years of age. ${ }^{2}$ India is rapidly becoming the "diabetes capital" of the world with over 300 million diabetic patients. ${ }^{3}$ What is more worrying is the realization that life style changes are beginning to transform type $2 \mathrm{DM}$ from a largely adult disorder into one that is affecting teenagers and children. ${ }^{4,5}$

Research on the negative health outcomes associated with diabetes has traditionally focused on the impact of metabolic dysregulation on cardiovascular disease and little consideration was given to the possibility that metabolic dysregulation might also influence brain function. However, things changed when researchers were forced to turn their attention to identify factors leading to the causation of Vascular Dementia, the second most common cause of Dementia. Not surprisingly, type 2 diabetes emerged as an aetiology for vascular dementia along with hypertension, stroke and atherosclerosis.6,7 Since then, evidence began to emerge showing the positive association between diabetes and cognitive impairment. Many overlapping mechanisms for this association have been proposed. Chronic hyperglycaemia and the production of advanced glycated end products may damage vascular tissue and endothelium, deoxy- ribonucleic acid (DNA) and mitochondria in the brain and increase free radicals, inflammatory responses and amyloid deposition. 8,9

However, type $2 \mathrm{DM}$ is a complex disorder and evaluation of cognitive dysfunctions associated with it is challenging because of the co-occurrence of confounding variables. Firstly, in addition to intrinsic abnormalities such as hyperglycaemia, patients with type $2 \mathrm{DM}$ frequently develop multisystem complication such as retinopathy, nephropathy and neuropathy, all of which interfere with an individual's performance on psychometric testing.

Secondly, patients with type 2 DM treated with insulin or sulfonylureas may also develop recurrent episodes of hypoglycaemia that are arguably associated with modest permanent cognitive dysfunctions. Finally, disorders such as hypertension, ischemic heart disease, cardiovascular disease, hyperlipidaemia and depression can cause cognitive dysfunctions themselves and are often co-morbid with type 2 DM. Thus, the cognitive impairment observed in type 2 DM may result from the interactions between abnormalities intrinsic to diabetes, diabetes specific complications and other diabetes related disorders. The above caveats and the observations from few studies which did not find any association between diabetes and cognitive functions, made it abundantly clear that impact of type 2 diabetes on cognitive functions is complex. Soon it became obvious that type 2 diabetes mellitus did not affect all subset of cognitive functions uniformly. Some cognitive functions are relatively spared while others are significantly affected. Certain set of cognitive functions are affected early while others are adversely impacted only later. For example, the use of MMSE for cognitive function assessment has shown conflicting results. ${ }^{10,11,12}$ On the other hand, studies which used measures of attention, psychomotor speed and visuomotor functions were more consistent in showing cognitive impairements. ${ }^{13,14,15,16,17}$

The current study is done in this context. This study attempts to find out cognitive dysfunctions intrinsic to diabetes. It focuses on attention, sequencing, visuomotor coordination, psychomotor speed and motor persistence; those set of cognitive functions which are more vulnerable to the adverse effects of type diabetes on the brain and may be affected earlier.

We wanted to determine and compare the psychomotor speed and visuomotor coordination functions in type 2 diabetes mellitus patients and in non-diabetics matched for age, education and socio-economic status.

\section{METHODS}

The data for this research paper is derived from a study on Cognitive Dysfunctions in type 2 diabetes mellitus and followed a cross sectional comparative design. It was done at Fr. Muller Medical College, a tertiary care teaching hospital located in the coastal city of Mangalore. Institutional ethical committee approval was obtained. Convenience sampling was used to select 30 patients who were diagnosed as having type 2 diabetes and they formed cases group. 30 non-diabetic controls were selected from relatives of the patients admitted under various specialties. Both cases and controls were matched for age, gender, education and socio-economic status.

Subjects fulfilling the inclusion and exclusion criteria were identified. An informed consent was obtained from those who were willing to participate in the study. The purpose of the study was explained to the participants. A specially designed sociodemographic and clinical data proforma was used to collect data of the study subjects. Psychomotor speed and visuomotor coordination of study subjects were assessed using Trail Making Test - Part B and Digit Symbol Substitution Test. Investigations like hemogram, random blood sugar, fasting blood sugar, post prandial blood sugar, glycosylated haemoglobin, lipid profile, liver function tests, renal function tests and ECG which were available were recorded.

Inclusion criteria for cases were as follows. Patients with type 2 diabetes as diagnosed by the Department of Internal Medicine based on blood sugar estimations as per World Health Organisation recommendation of Fasting Blood Sugar $\geq$ $126 \mathrm{mg} / \mathrm{dl}$ and 2-hour post prandial blood sugar $\geq 200 \mathrm{mg} / \mathrm{dl}$. The age of the subjects should be between 30-65 years and must have passed $8^{\text {th }}$ standard.

Exclusion criteria for the cases took into consideration, the effect of age and medical comorbidities on cognitive function. Hence, type 2 diabetics more than 65 years of age and those with comorbid hypertension, history of neurodegenerative diseases like dementia and organic mental disorders were excluded. Those patients with history of substance abuse, with past or current history of psychiatric disorders were also excluded.

Subjects aged $30-65$ years with minimum $8^{\text {th }}$ standard education without past or current diagnosis of type 2 DM formed the control group. Patients with chronic diseases which may cause cognitive impairment such as hypertension, neurodegenerative diseases like dementia and organic mental 
disorders, those with history of substance abuse, with past or current history of psychiatric disorders were excluded. The cognitive functions which were the focus of this study namely psychomotor speed and visuomotor coordination was assessed using Trail Making Test-Part B and Digit Symbol Substitution Test. A brief description of these two tests is provided in the following sections.

\section{Trial Making Test Part B (TMT-B) ${ }^{18,19,20}$}

TMT, originally constructed as "Partington's Pathways" or "Divided Attention Test" is a timed test of speed for attention, sequencing, mental flexibility, visual search and motor function. TMT is available in various formats namely TMTPart A and TMT Part B, Oral Trail Making test and Colour Trails test. TMT- Part A requires connection, by making pencil lines between 25 encircled numbers randomly arranged, in a proper order while Part B has 25 encircled numbers and letters in alternating order. Scoring is expressed in terms of time in seconds required to complete the test. If an error is made, the examiner will point it out to the patient for correction and have them return to and continue from the correct location while the clock remains running. Errors are recorded and the subject continues with the test. Scores are strongly influenced by age, education and intelligence of the subjects. Interpretations of scores are based on normative data. TMT Part-B is associated with the processes of distinguishing between numbers and letters, integration of two independent series, ability to learn an organizing principle and apply it systematically, reveal retention and integration, verbal problem solving and planning.

\section{Digit Symbol Substitution Test 21}

It is a test of visuomotor co-ordination, motor persistence, sustained attention and response speed. Rapid information processing is required in order to substitute the symbols accurately and quickly. The test consists of a sheet in which number 1-9 are randomly arranged in 4 rows of 25 squares each. The subjects substitute each number with a symbol using a number symbol key given at the top of the page. The time taken to complete the test in seconds forms the score and errors made are noted down.

The data was analysed for statistical significance by using chi- square test for categorical variables, ANOVA, Student's ttest and Mann - Whitney $U$ test for continuous numerical variables. Carl Pearson's coefficient of correlation was used to find out the correlation between the variables.

\section{RESULTS}

There is no significant difference between the age of control group and cases. Significant difference between the mean BMI value of the controls and cases $(\mathrm{p}=0.012<0.05)$ was observed which indicated that diabetics were significantly overweight with respect to their nondiabetic counterparts. The mean random blood sugar value in the cases was more than twice the mean value in the control group which was very highly significant $(\mathrm{p}=0.001<0.01)$.

On the measures of psychomotor speed and visuomotor functions there were significant differences between the two groups. The diabetic patients not only took more time to complete both TMT-B and DSST, they made more errors as well compared to their nondiabetic counterparts. The data is given in tables. Not surprisingly, the number of errors had an adverse impact on the total time taken to complete the test.

TMTT also had a statistically significant negative association with $\mathrm{HbA} 1 \mathrm{c}(\mathrm{p}=0.047<0.05)$. Results indicated that longer the duration of diabetes, greater was the impairment in the psychomotor functions. There was a very highly significant positive correlation of TMTT with DSSTT $(p=0.000<0.001)$. TMTE was very highly significantly positive correlation with DSSTE $(\mathrm{p}=0.000<0.001)$.

\begin{tabular}{|c|c|c|c|c|c|}
\hline & Group & $\mathbf{N}$ & Mean & Std. Deviation & $\mathbf{Z}^{*}$ \\
\hline \multirow{2}{*}{ RBS } & Control & 30 & 112.2000 & 9.21543 & 5.4121 \\
\cline { 2 - 5 } & Cases & 30 & 278.1667 & 89.91724 & $\mathrm{p}=0.001 \mathrm{vhs}$ \\
\hline \multicolumn{6}{|c|}{ Table 1. Random Blood Sugar } \\
\hline
\end{tabular}

\begin{tabular}{|c|c|c|c|c|}
\hline & Group & Mean & Std. Deviation & $\mathbf{Z}^{*}$ \\
\hline \multirow{2}{*}{ TMTT $^{+}$} & Control & 144.6667 & 27.41549 & $\mathrm{Z}=4.562$ \\
& Case & 264.1667 & 75.12155 & $\mathrm{p}=0.001$ vhs \\
\hline DSSTT $^{*}$ & Control & 285.1667 & 59.22609 & $\mathrm{Z}=5.92$ \\
& Case & 531.8333 & 86.17828 & $\mathrm{p}=0.001$ vhs \\
\hline \multicolumn{5}{|c|}{ Table 2. Time Taken in Seconds on TMT-B and DSST } \\
\hline * Mann-Whitney U Test, + Trail Making Test Time, * Digit Symbol Substitution Test \\
Time
\end{tabular}

\begin{tabular}{|c|c|c|c|c|}
\hline & Group & Mean & Std. Deviation & t \\
\hline \multirow{2}{*}{ TMTE $^{*}$} & Control & 1.7000 & 0.91539 & 16.65100 \\
& Case & 6.8667 & 1.43198 & $\mathrm{p}=0.001 \mathrm{vhs}$ \\
\hline \multirow{2}{*}{ DSSTE $^{+}$} & Control & 2.4000 & 1.16264 & 12.93100 \\
& Case & 8.2667 & 2.19613 & $\mathrm{p}=0.001 \mathrm{vhs}$ \\
\hline \multicolumn{5}{|c|}{ Table 3. No. of Errors in TMT-B and DSST } \\
\hline${ }^{*}$ Trail Making Test Errors, ${ }^{+}$Digit Symbol Substitution Test Errors \\
\hline
\end{tabular}

\begin{tabular}{|c|c|c|c|c|c|}
\hline & $\begin{array}{l}\text { Duration } \\
\text { in Years }\end{array}$ & $\begin{array}{l}\text { Mean } \\
\text { Score } \\
\end{array}$ & $\begin{array}{c}\text { Std. } \\
\text { Deviation }\end{array}$ & $\mathbf{F}$ & $\mathbf{p}$ \\
\hline \multirow{3}{*}{ TMTT } & $<5$ & 150.6579 & 28.71507 & \multirow{3}{*}{141.382} & \multirow{3}{*}{0.001 vhs } \\
\hline & $5-10$ & 265.0000 & 52.83622 & & \\
\hline & $>10$ & 343.8889 & 19.49003 & & \\
\hline \multirow{3}{*}{ DSSTT } & $<5$ & 321.8421 & 91.17418 & \multirow{3}{*}{58.002} & \multirow{3}{*}{0.001 vhs } \\
\hline & $5-10$ & 520.0000 & 61.13510 & & \\
\hline & $>10$ & 613.3333 & 81.20191 & & \\
\hline \multirow{3}{*}{ TMTE } & $<5$ & 2.7895 & 2.33837 & \multirow{3}{*}{26.797} & \multirow{3}{*}{$0.001 \mathrm{vhs}$} \\
\hline & $5-10$ & 6.5385 & 1.33012 & & \\
\hline & $>10$ & 7.3333 & 1.80278 & & \\
\hline \multirow{3}{*}{ DSSE } & $<5$ & 3.7105 & 2.94919 & \multirow{3}{*}{18.895} & \multirow{3}{*}{0.001 vhs } \\
\hline & $5-10$ & 7.7692 & 2.00640 & & \\
\hline & $>10$ & 8.6667 & 2.44949 & & \\
\hline \multicolumn{6}{|c|}{ Table 4. Duration of diabetes Vs Cognitive Tests (Cases) } \\
\hline
\end{tabular}

\section{DISCUSSION}

Screening for cognitive dysfunctions in general population became popular and efficient with the advent of screening instruments such as Mini Mental State Examination (MMSE). These instruments were easy to use, were inexpensive and could be taught to primary care physicians and volunteers. Moreover, translation of MMSE in various languages is widely available around the world.

Unfortunately, the very advantages of screening instruments; the brevity and ease of use begin to work against them when more nuanced look at cognitive functions are required. Here it may be worthwhile to ponder what the term cognitive function stands for in its truest sense. It refers to the highest levels of various mental processes such as perception, memory, abstract thinking and reasoning and problem solving as well as the more integrative and control process related to executive functions such as planning, choosing strategies and the enactment of these strategies. 
Evidently, MMSE and similar screening instruments are biased toward verbal items and does not adequately measure other cognitive functions such as ability to attend to relevant input, ability to solve abstract problems, psychomotor speed and visuospatial ability, cognitive functions that may be the ones, first to be affected by diabetes. ${ }^{13,22,23}$

What are the implications of this when we study cognitive dysfunctions in a disease like type 2 diabetes mellitus? It means that cognitive impairments likely to occur in diabetes may go undetected especially when they are subtle. Domainspecific tests which probe the blind areas of screening cognitive instruments are necessary to fill this void. Current study employed two domain specific tests, TMT-B and DSST to comprehensively assess cognitive function, a notable strength of the study. Cognitive tests were administered to the subjects within 2 hours after the last meal ingestion. This precaution was taken because type 2 DM patients are prone to develop discrete episodes of hypoglycaemia, as an adverse effect of medications prescribed to achieve normoglycemia. Since hypoglycaemia is known to affect cognitive functions adversely, cognitive testing during such hypoglycaemic episodes might lead to erroneous interpretation of results.

On TMT-B, diabetic group took nearly twice the time consumed by the control group to complete the test. Diabetic group also made more number of errors - four times as the control group. These findings are in agreement with results from earlier studies. ${ }^{10,13,15,16}$ TMT-B may be more sensitive to cognitive decline than MMSE, which was primarily designed to screen for dementia than measure cognitive function. The use of TMT-B, may help to detect the presence of preclinical cognitive decline and circumvent the ceiling effects of MMSE, a methodological strong point of this study.

DSST, a timed test of attention, psychomotor performance and perceptual organization was adversely affected by diabetes and difference between the control group and patients with diabetes was very highly significant. The study by Ryan et $\mathrm{al}^{13}$ found that in middle-aged diabetics, learning and verbal memory were unaffected while psychomotor slowing was marked as reflected by impaired performance on DSST. It has been employed in a number of studies and in each of these studies, diabetics took significantly longer time and made more number of errors while completing the test. $10,14,15,17,24$

One compelling explanation for the impairments noted in TMT-B and DSST is due to the psychomotor slowing seen in type 2 diabetic patients which may involve the polyol pathway. Glucose is ordinarily converted to fructose by sorbitol dehydrogenase. The intra-cellular accumulation of sorbitol and fructose leads to a depletion of myoinositol which in turn is associated with a reduction in $\mathrm{Na}^{+} / \mathrm{K}^{+}$ATPase activity in plasma membranes. This affects intracellular metabolism and leads to a subsequent reduction in peripheral nerve conduction efficiency leading to peripheral neuropathy and the resultant psychomotor slowness. ${ }^{23}$

There was statistically significant difference $(p=$ $0.012<0.05$ ) between the body mass index of diabetics and non-diabetics in the current study. This was in agreement with results from other studies.10,25,26,27 However, there was an essential difference. The mean BMI (23.41) of diabetics in the current study was still with in normal limits (BMI: 18-24) unlike in the above cited studies in which BMI of diabetic patients was in the range of obesity. This assumes importance because Elias et al,28 from the Framingham investigation of obesity and cognitive function, identified obesity as a risk factor for cognitive dysfunction independently of diabetes, total cholesterol, alcohol consumption, cigarette smoking, hypertension and stroke. The fact that, in the current study, diabetic subjects were not obese eliminated a potential confounding factor and gave further credence to the hypothesis at the beginning of the study, i.e., cognitive deficits observed are due to the intrinsic effect of diabetes.

Another significant finding noted is that duration of diabetes was a very highly significant predictor of cognitive decline, in both the tests employed. In TMT-B, the time taken to complete the test increased by more than two-fold in subjects with duration of diabetes greater than 10 years with respect to those having diabetes for less than 5 years and a similar trend was noticed in DSST as well. Longer the duration of type $2 \mathrm{DM}$, greater was the number of errors made, in TMT$\mathrm{B}$ as well as DSST which was statistically very highly significant. Similar conclusions were drawn by earlier studies. ${ }^{15,16,29}$

Glycosylated $\mathrm{Hb}$ level was inversely correlated with performance in TMT-B. Possible explanations for this include the hyperinsulinaemic state induced by the chronic use of insulin and the hypoglycaemic episodes which are more likely to occur with insulin use than OHA use. Other reasons for this association might be the fact that, patients taking insulin would have had longer duration of diabetes, more severe illness and are more aged.

However, there are certain limitations to this study. Current study had a cross-sectional design. Performance at a single point may bear little relation to subsequent changes in performance over time and hence the ability to infer change from a single observation is limited. A causal relationship cannot be established from a cross-sectional study because a cross-sectional relationship may be a result of associations with a third variable in common with both the risk factor and cognitive function rather than a true cause and effect.

Since cognition is a measure of change in an individual over time, longitudinal studies are more reliable. Depression, a common comorbidity of type 2 DM can confound the results. In this study depression was ruled out basing on careful clinical examination but no objective or subjective rating scales were used.

\section{CONCLUSIONS}

The impact of type 2 diabetes mellitus on the genesis and pattern of cognitive deficits is complex. It appears from this study that cognitive functions of attention, sequencing, visuomotor coordination, psychomotor speed and motor persistence are affected adversely by type 2 diabetes mellitus and this is correlated with advancing age and longer duration of poorly controlled diabetes. Confirmation of findings from this study with longitudinal studies is required because prospective studies can link risk factors with direct measures of change in cognitive function over time. They can greatly reduce bias, will allow more definitive conclusions to be drawn regarding cause and effect, and will markedly increase study power. Finally, changes in cognitive ability in old age are brought about both by processes of 'normal' ageing and by a 
steadily accumulating burden of pathologies. The main task of cognitive gerontology is to compare the relative contributions of these two factors and their interactions. The study of various subsets of cognitive functions in type 2 diabetes mellitus provides us with great insights into these complex interactions.

\section{ACKNOWLEDGEMENT}

The author thanks Dr. K. Krishnamurthy, Former Professor and Head of the Department of Psychiatry of Fr. Muller Medical College at the time of the study for his guidance and encouragement.

\section{REFERENCES}

[1] Barnett DM, Krall LP. The History of diabetes. In: Kahn CR, Weir GC, King GL, et al. eds. Joslin's diabetes mellitus. $14^{\text {th }}$ edn. Noida: Lippincott Williams \& Wilkins 2005: p. 1-17.

[2] Powers AC. Diabetes mellitus. In: Kasper DL, Braunwald E, Fauci AS, et al. eds. Harrison's Principles of Internal Medicine. $16^{\text {th }}$ edn. New Delhi: McGraw-Hill Publications 2005: p. 2152-80.

[3] Ramachandran A, Das AK. Basic considerations of diabetes mellitus. In: Shah SN, Anand MP, Acharya VN, et al. eds. API Textbook of Medicine. $7^{\text {th }}$ edn. Mumbai: The Association of Physicians of India 2003 : p. 1097-8.

[4] Kitagawa $T$, Owada $M$, Urakami $T$, et al. Increased incidence of non-insulin dependent diabetes mellitus among Japanese schoolchildren correlates with an increased intake of animal protein and fat. Clin Pract 1998;37(2):111-6.

[5] Bloomgarden ZT. Type 2 diabetes in the young. The evolving epidemic. Diabetes Care 2004;27(4):998-1010.

[6] Ott A, Stolk RP, Hofman A, et al. Association of diabetes mellitus and dementia: The Rotterdam Study. Diabetologia 1996;39(11):1392-7.

[7] MacKnight C, Rockwood K, Awalt E, et al. Diabetes mellitus and the risk of dementia, Alzheimer's disease and vascular cognitive impairment in the Canadian study of health and aging. Dement Geriatr Cogn Disord 2002;14(2):77-83.

[8] Strachan MWJ, Deary IJ, Ewing FM, et al. Is type II diabetes associated with an increased risk of cognitive dysfunction? A critical review of published studies. Diabetes Care 1997;20(3):438-45.

[9] Gregg EW, Brown A. Cognitive and physical disabilities and aging-related complications of diabetes. Clinical diabetes 2003;21(3):113-7.

[10] Fontbonne A, Berr C, Ducimetiere P, et al. Changes in cognitive abilities over a 4- year period are unfavorably affected in elderly diabetic subjects: results of the Epidemiology of Vascular Aging Study. Diabetes Care 2001;24(2):366-70.

[11] Muquit MM, Ferdous HS. Cognitive impairment in elderly, non-insulin dependent diabetic men in Bangladesh. Bangladesh Med Res Counc Bull 1998;24(2):23-6.
[12] Lindeman RD, Romero LJ, LaRue A, et al. A Biethnic community survey of cognition in participants with type 2 diabetes, impaired glucose tolerance and normal glucose tolerance. The New Mexico Elder Health Survey. Diabetes Care 2001;24(9):1567-72.

[13] Ryan CM, Williams TM, Orchard TJ, et al. Psychomotor slowing is associated with distal symmetrical polyneuropathy in adults with diabetes mellitus. Diabetes 1992;41(1):107-13.

[14] Mogi N, Umegaki H, Hattori A, et al. Cognitive function in Japanese elderly with type 2 diabetes mellitus. J diabetes Complications 2004;18(1):42-6.

[15] Gregg EW, Yaffe K, Cauley JA, et al. Is diabetes associated with cognitive impairment and cognitive decline among older women? Arch Intern Med 2000;160(2):174-80.

[16] Yaffe K, Blackwell T, Kanaya AM, et al. Diabetes, impaired fasting glucose and development of cognitive impairment in older women. Neurology 2004;63(4):658-63.

[17] Knopman D, Boland LL, Mosley T, et al. Cardiovascular risk factors and cognitive decline in middle aged adults. Neurology 2001;56(1):42-8.

[18] Tombaugh TN. Trail Making Test A and B: Normative data stratified by age and education. Arch Clin Neuropsychol 2004;19(2):203-14.

[19] Spreen O, Strauss E. Visual, visuomotor and auditory tests. In: A compendium of neuropsychological tests: administration, norms and commentary. $2^{\text {nd }}$ edn. New York: Oxford University Press 1998: p. 533-47.

[20] Greenlief CL, Margolis RB, Erker GJ. Application of the Trail Making Test in differentiating neuropsychological impairment of elderly persons. Percept Mot Skills 1985;61(3 Pt 2):1283-9.

[21] Rao SL, Subbakrishna DK, Gopukumar K. NIMHANS neuropsychological battery-2004 Manual. $1^{\text {st }}$ edn. Bangalore: NIMHANS Publications 2004: p. 11-17.

[22] Ryan CM, Geckle MO. Circumscribed cognitive dysfunction in middle-aged adults with type 2 diabetes. Diabetes Care 2000;23(10):1486-93.

[23] Ryan CM. Diabetes, aging and cognitive decline. Neurobiol Aging 2005;26(Suppl 1):S21-5.

[24] Perlmuter LC, Tun P, Sizer N, et al. Age and diabetes related changes in verbal fluency. Exp Ageing Res 1987;13(1-2):9-14.

[25] Vanhanen M, Koivisto K, Kuusisto J, et al. Cognitive function in an elderly population with persistent impaired glucose tolerance. Diabetes Care 1998;21(3):398-402.

[26] Sommerfield AJ, Deary IJ, Frier BM. Acute hyperglycemia alters mood state and impairs cognitive performance in people with type 2 diabetes. Diabetes Care 2004;27(10):2335-40.

[27] Greenwood CE, Kaplan RJ, Hebblethwaite S, et al. Carbohydrate induced memory impairment in adults with type 2 diabetes. Diabetes Care 2003;26(7):1961-6.

[28] Elias MF, Elias PK, Sullivan LM, et al. Obesity, diabetes and cognitive deficit: The Framingham Study. Neurobiol Aging 2005;26(Suppl 1):S11-S6.

[29] Cosway R, Strachan MW, Dougall A, et al. Cognitive functioning and information processing in type 2 diabetes. Diabet Med 2001;18(10):803-10. 